Supporting Information

\title{
Efficient Red Phosphorescent Polymers with Trap-Assisted Charge \\ Balance: Molecular design, Synthesis and Electroluminescent Properties
}

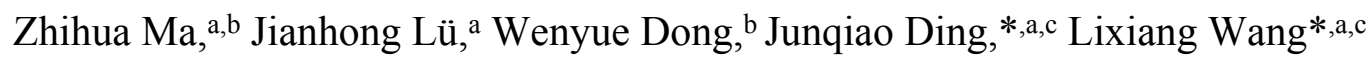

a State Key Laboratory of Polymer Physics and Chemistry, Changchun Institute of Applied Chemistry, Chinese Academy of Sciences, Changchun 130022, P. R. China

${ }^{b}$ School of Materials Science and Engineering, Changchun University of Science and Technology, Changchun 130022, P. R. China

${ }^{c}$ University of Science and Technology of China, Hefei 230026, P. R. China

\section{Corresponding Author}

* E-mail: junqiaod@ciac.ac.cn (Junqiao Ding), lixiang@ciac.ac.cn (Lixiang Wang) 

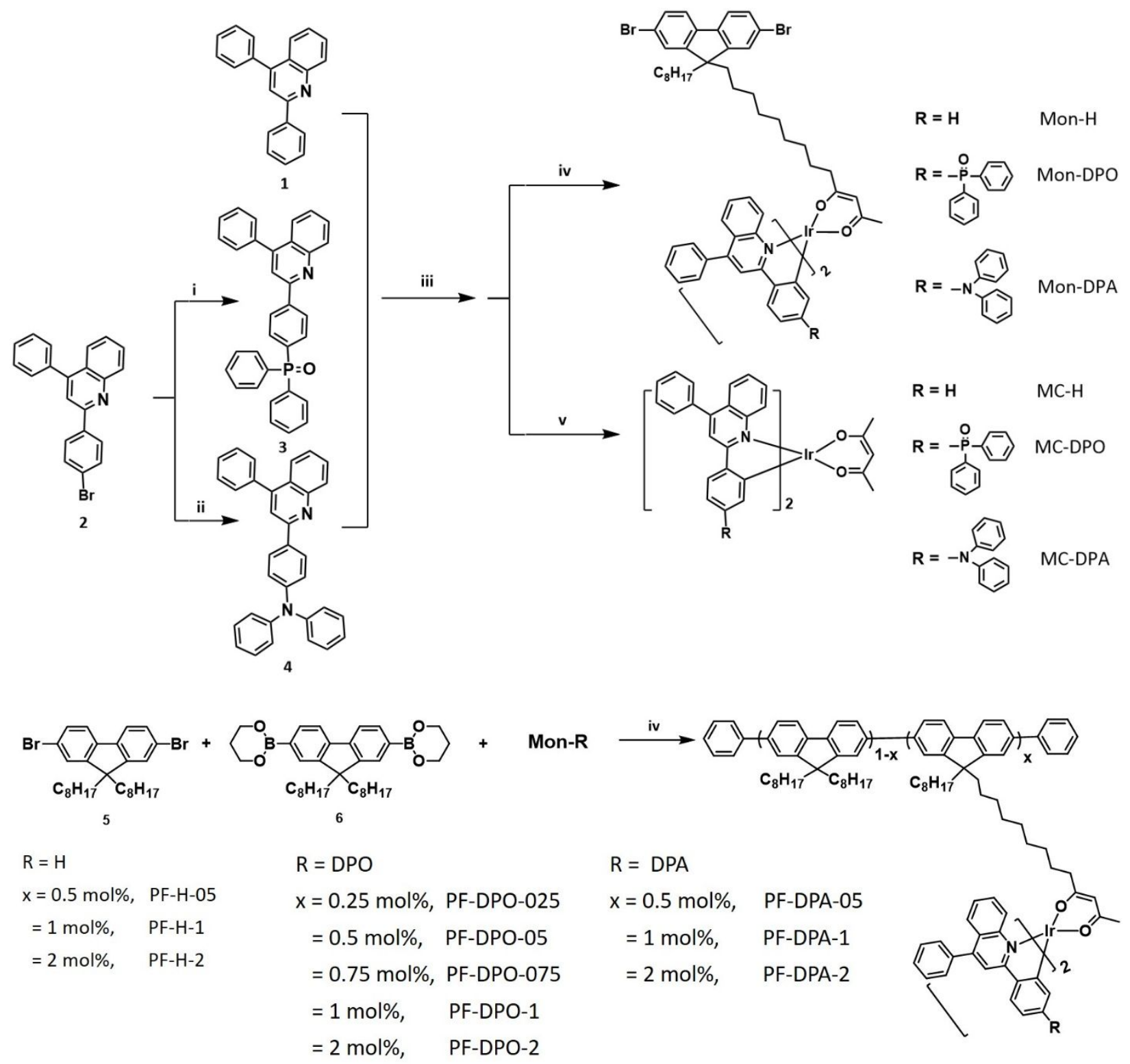

Scheme S1. Synthetic routes of model Ir complexes and the phosphorescent polymers. Reagents and conditions: i) $n$-BuLi, THF, $-78{ }^{\circ} \mathrm{C}$; chlorodiphenylphosphine, THF, $-78{ }^{\circ} \mathrm{C} ; 30 \% \mathrm{H}_{2} \mathrm{O}_{2}$, dichloromethane; ii) diphenylamine, $\mathrm{Pd}(\mathrm{OAc})_{2}$, tri-tert-butylphosphine, $t$-BuONa, toluene, $100{ }^{\circ} \mathrm{C}$; iii) $\mathrm{IrCl}_{3} \cdot 3 \mathrm{H}_{2} \mathrm{O}$, ethoxyethanol/water, reflux; iv) DBrFacac, $\mathrm{Na}_{2} \mathrm{CO}_{3}$, acetonitrile/chloroform, reflux; v) acetyl acetone, $\mathrm{Na}_{2} \mathrm{CO}_{3}$, ethoxyethanol, reflux; iv) $\mathrm{Pd}\left(\mathrm{PPh}_{3}\right)_{4}$, potassium carbonate, aliquat 336 , toluene/water, $90{ }^{\circ} \mathrm{C}$. 
(a)

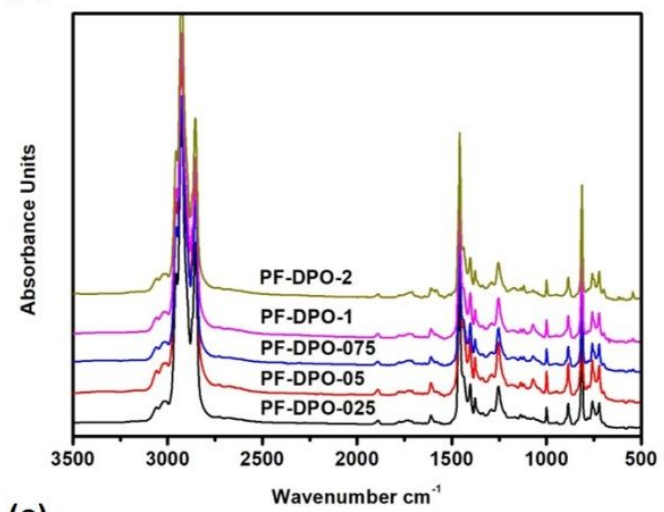

(c)

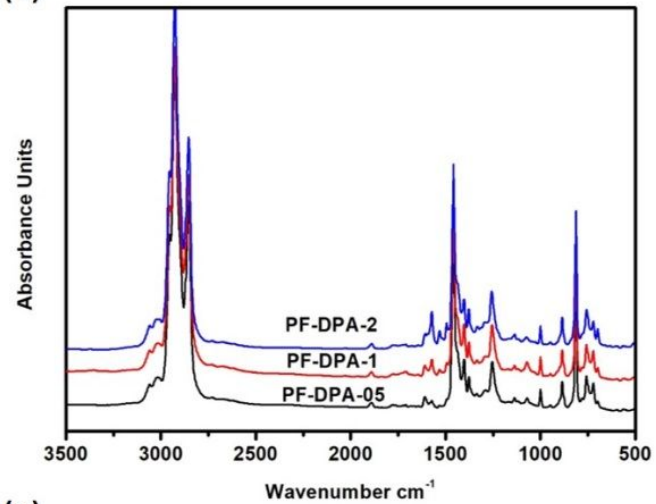

(e)

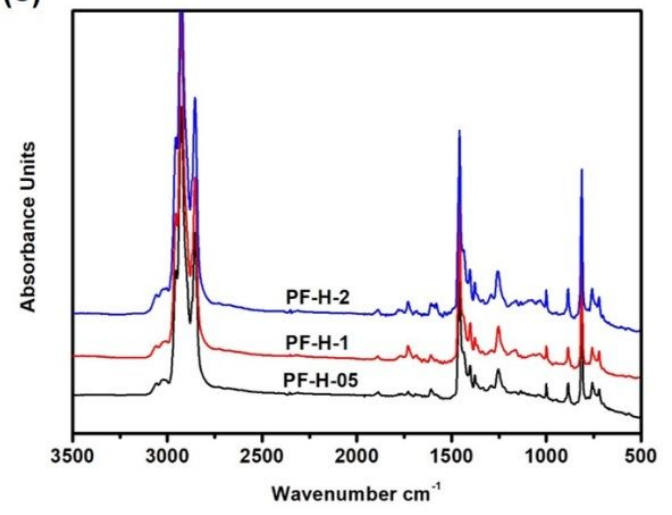

(b)

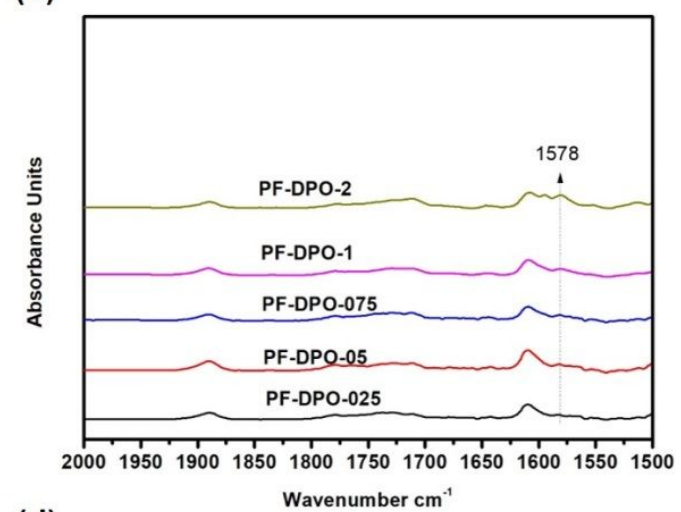

(d)

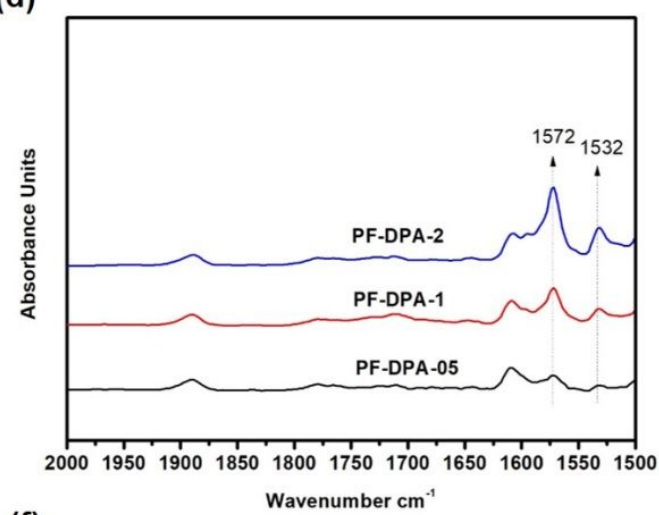

(f)

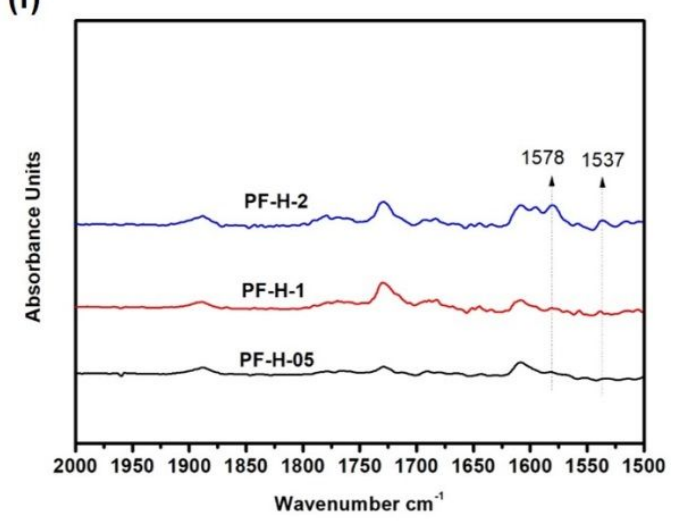

Figure S1. Full and partial (wavenumber from 2000 to $1500 \mathrm{~cm}^{-1}$ ) IR spectra of the phosphorescent polymers (PF-DPO-x (a, b), PF-DPA-x (c, d) and PF-H-x (e, f)). 


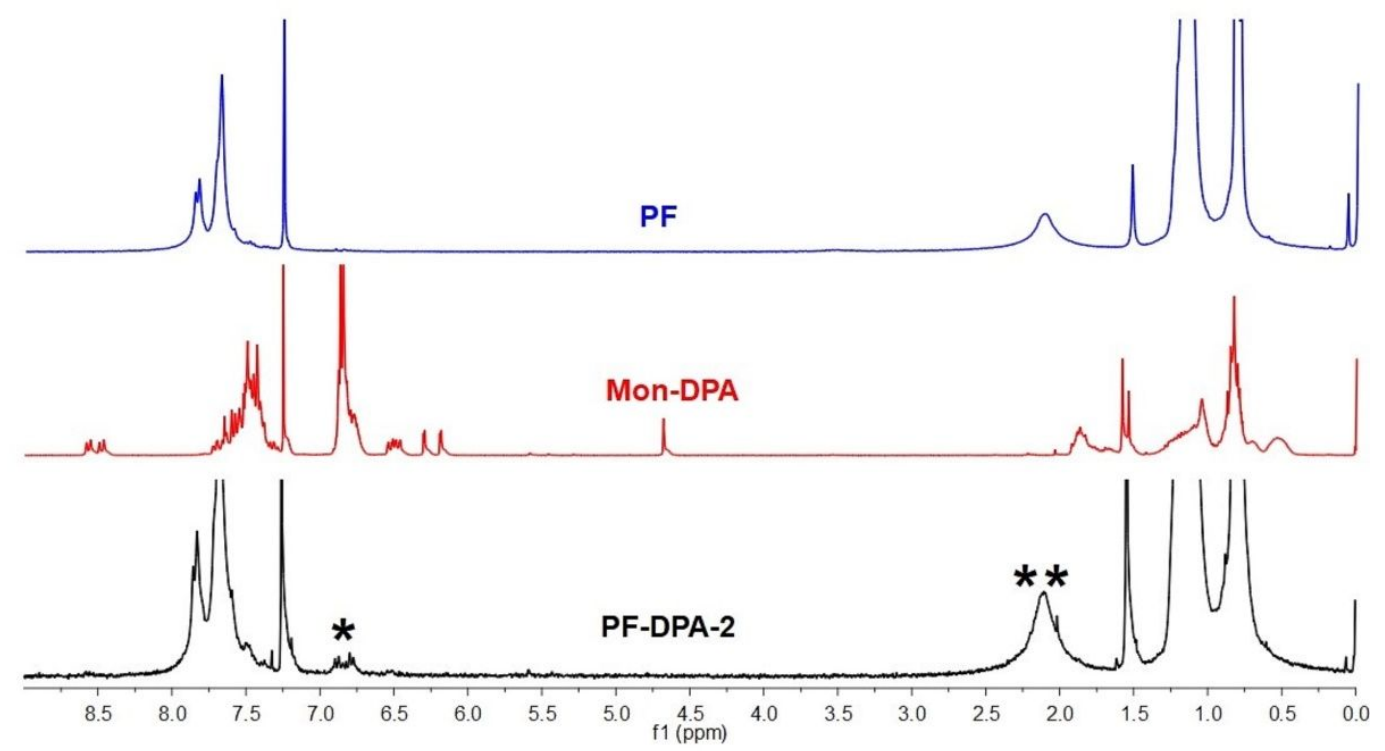

Figure S2. ${ }^{1} \mathrm{H}$ NMR spectra of PF, the monomer Mon-DPA and the polymer PF-DPA-2 (*: signals belong to the cyclometalated ligand of Ir complex; **: signals belong to the $\mathrm{CH}_{2}$ groups of the alkyl chains adjacent to the 9-position of fluorene). 
(a)
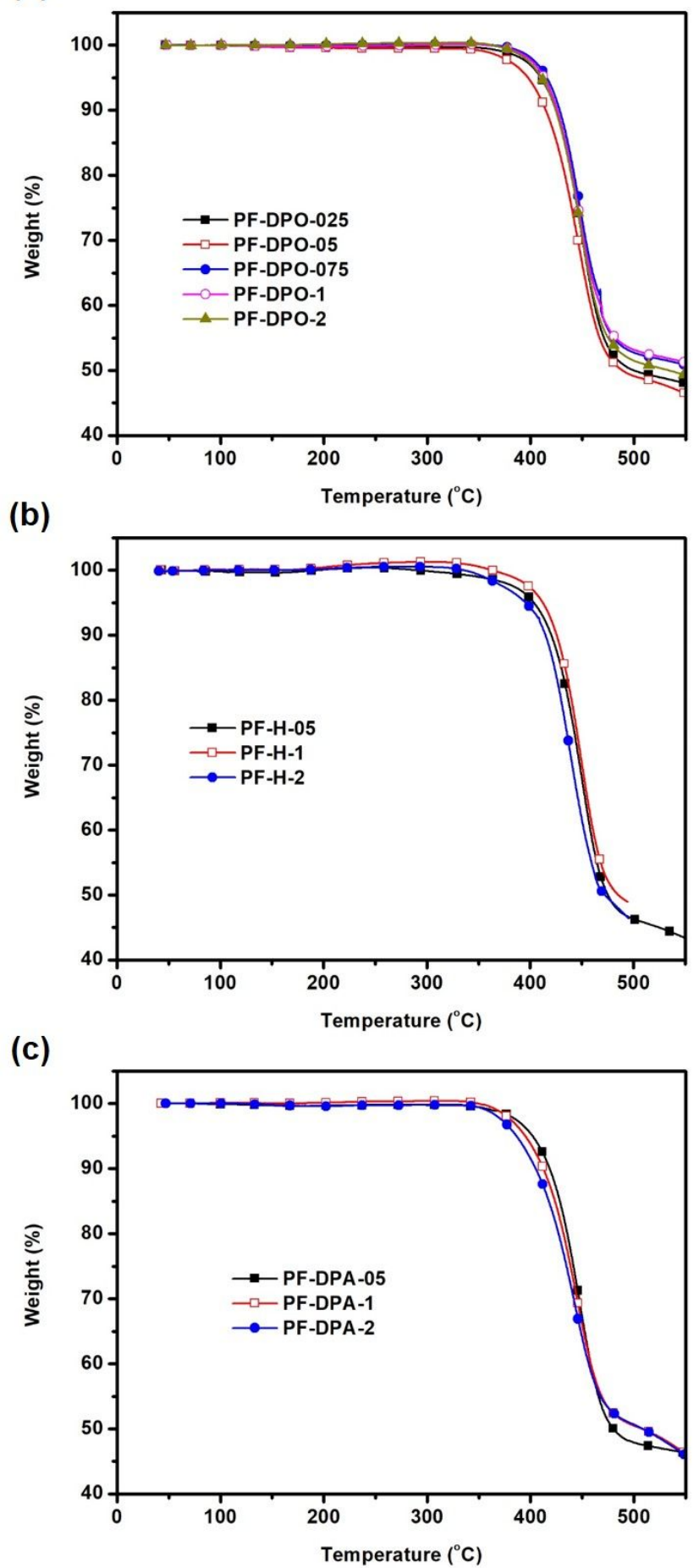

Figure S3. Thermogravimetic analysis (TGA) curves of PF-DPO-x (a), PF-H-x (b) and PF-DPA$x(c)$. 


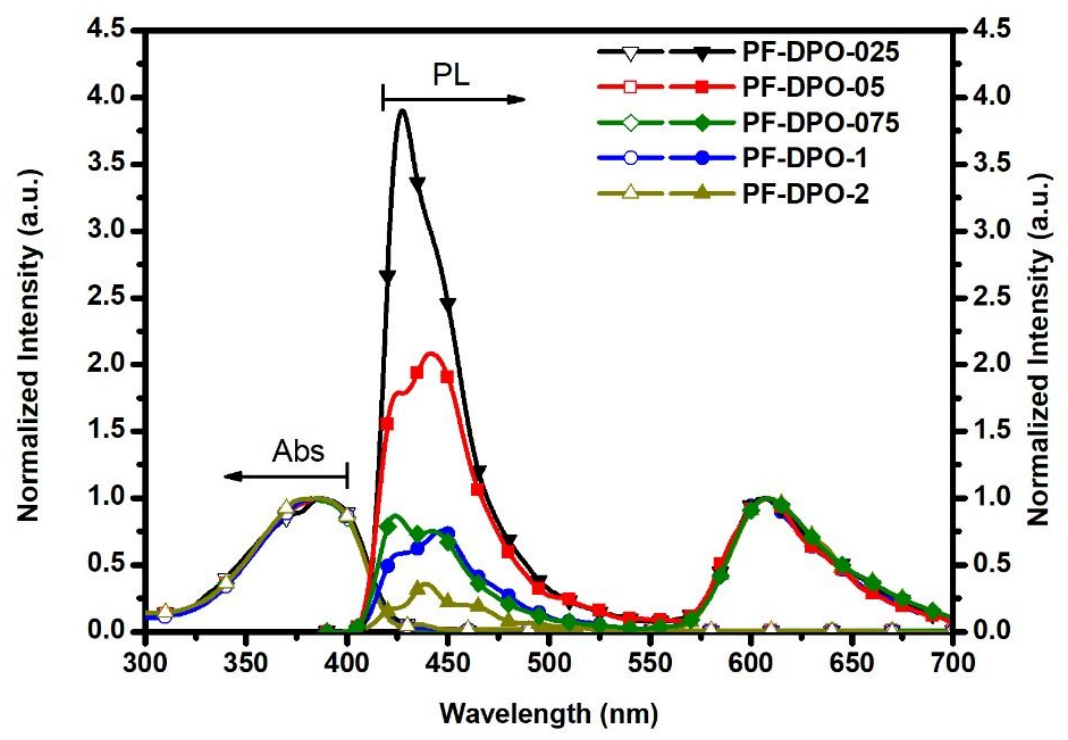

Figure S4. Absorption and PL spectra of PF-DPO-x in film state. 

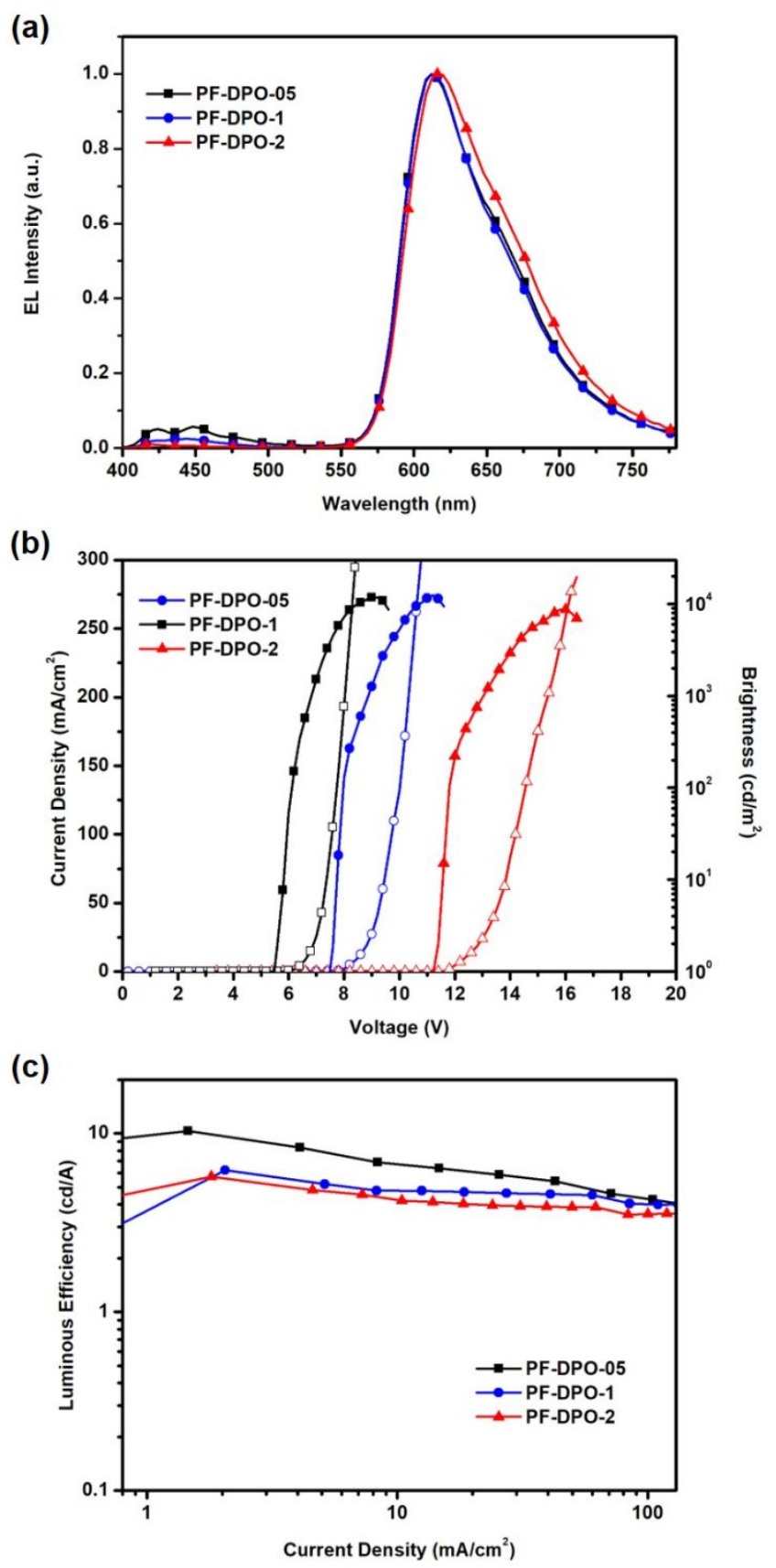

Figure S5. EL spectra (a), current density-voltage-luminescence characteristics (b, solid symbols for Brightness and hollow symbols for current density), current density dependence of the luminous efficiency (c) for PF-DPO-x. 

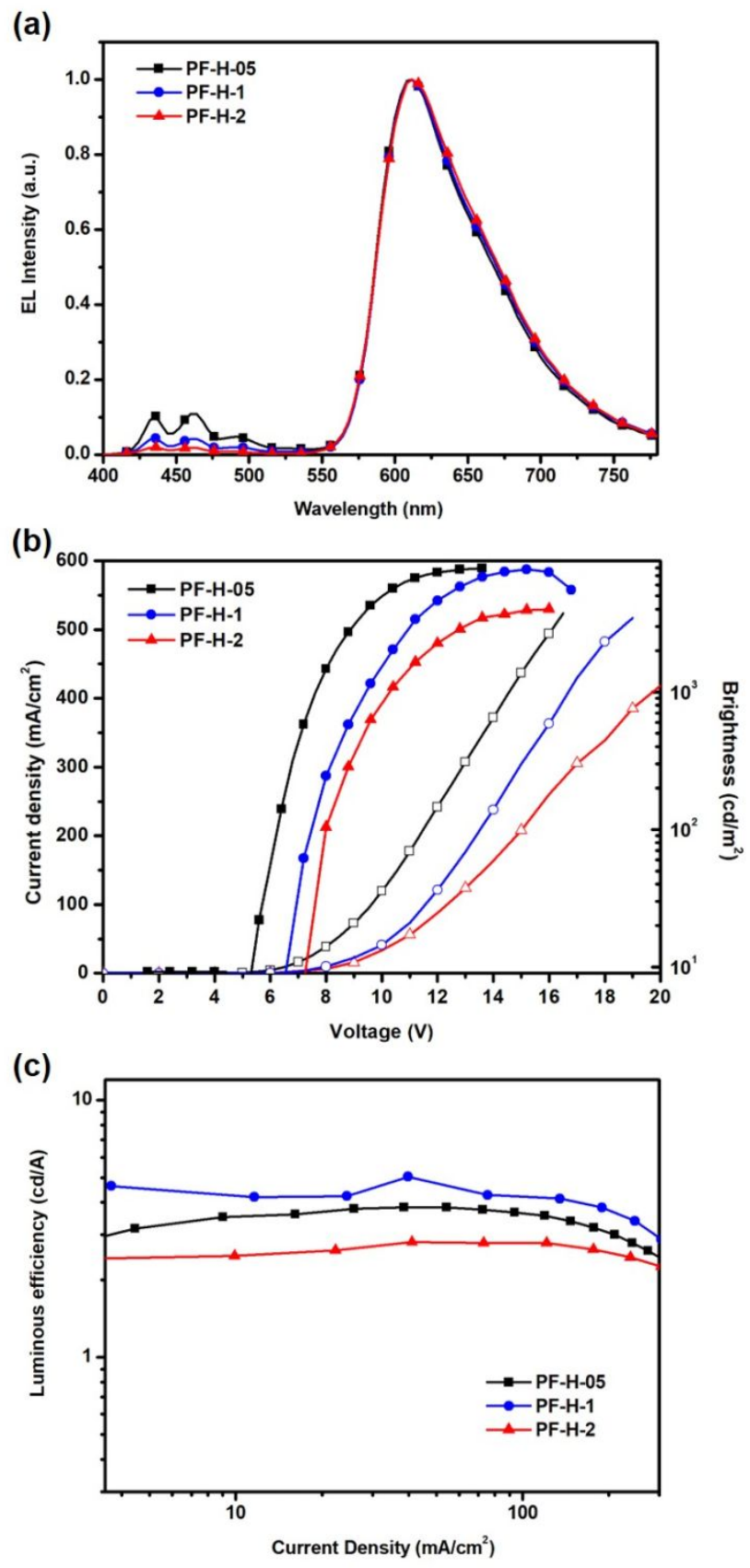

Figure S6. EL spectra (a), current density-voltage-luminescence characteristics (b, solid symbols for Brightness and hollow symbols for current density), current density dependence of the luminous efficiency (c) for PF-H-x. 


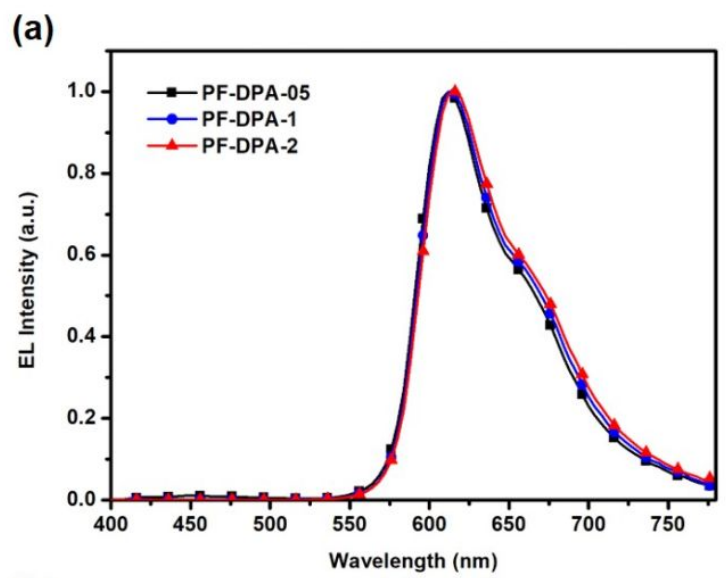

(b)
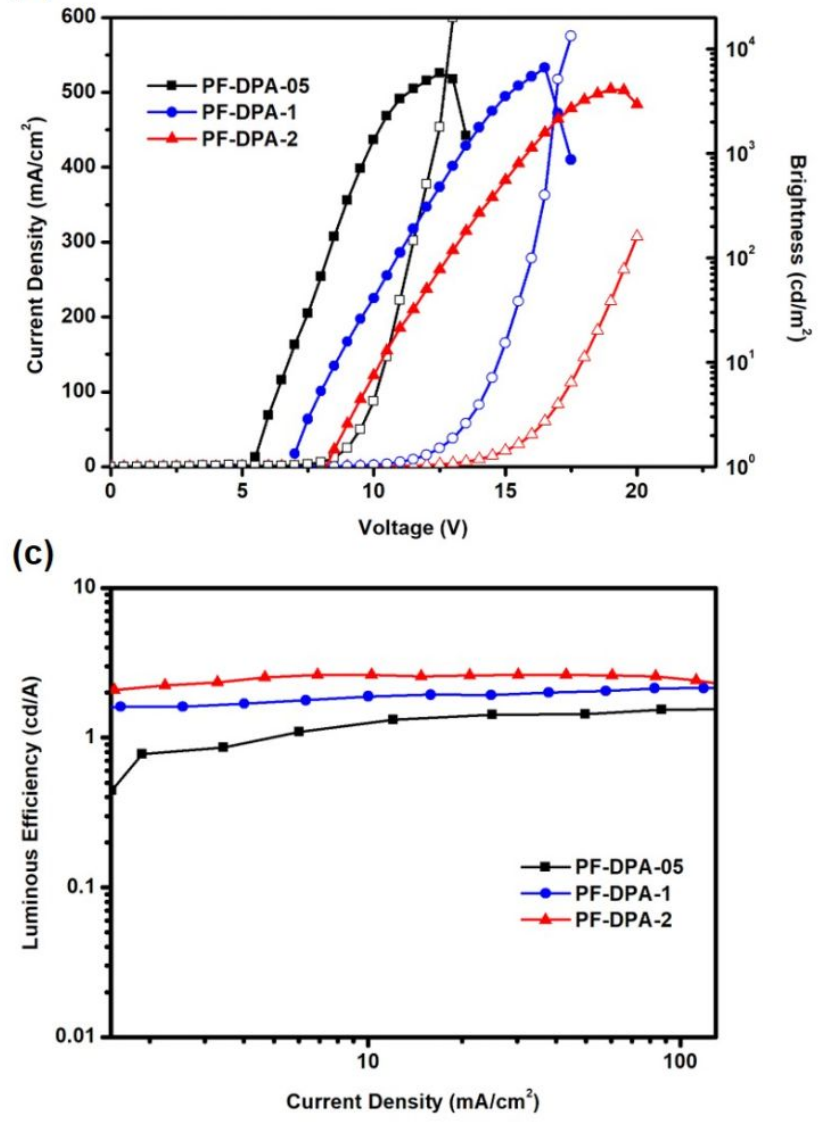

Figure S7. EL spectra (a), current density-voltage-luminescence characteristics (b, solid symbols for Brightness and hollow symbols for current density), current density dependence of the luminous efficiency (c) for PF-DPA-x. 
Table S1. Photophysical and electrochemical properties of the model complexes

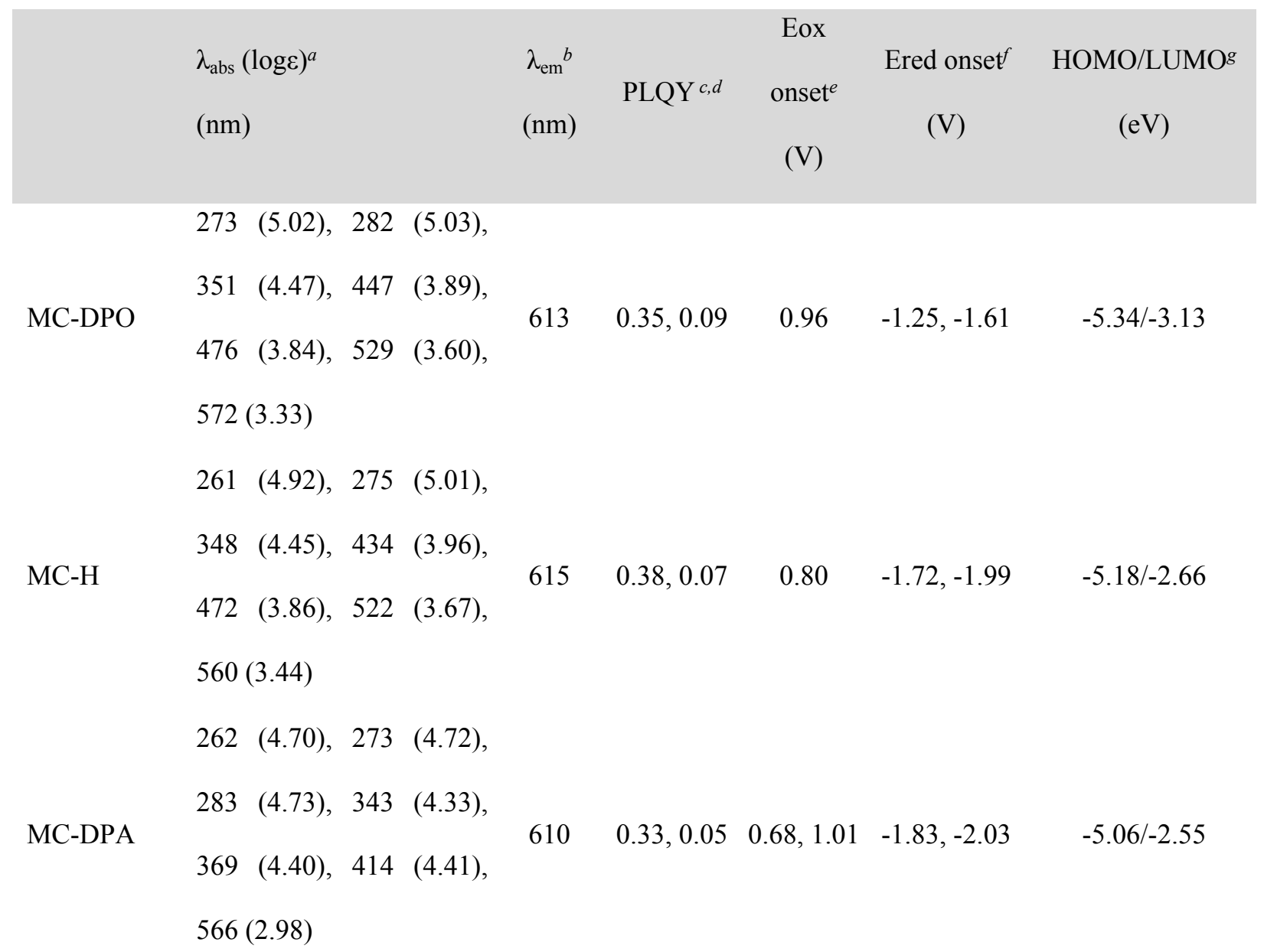

${ }^{a}$ Measured in aerated $\mathrm{CH}_{2} \mathrm{Cl}_{2}$ solutions $\left(10^{-5} \mathrm{M}\right)$ at $298 \mathrm{~K} .{ }^{b}$ Measured in aerated $\mathrm{CH}_{2} \mathrm{Cl}_{2}$ solutions $\left(10^{-5} \mathrm{M}\right)$ at $298 \mathrm{~K} .{ }^{c}$ Measured in degassed toluene solution by integrating sphere with excitation wavelength of $470 \mathrm{~nm} .{ }^{d}$ Measured in neat film by integrating sphere with excitation wavelength of $470 \mathrm{~nm} .{ }^{e}$ In $\mathrm{CH}_{2} \mathrm{Cl}_{2} \mathrm{vs} . \mathrm{Fc} / \mathrm{Fc}^{+} .{ }^{f}$ In THF vs. $\mathrm{Fc} / \mathrm{Fc}^{+} .{ }^{g} \mathrm{HOMO}=-\mathrm{e}(4.8 \mathrm{~V}+$ Eox onset $), \mathrm{LUMO}=-\mathrm{e}(4.8 \mathrm{~V}+$ Ered onset $)$. 


\section{Synthetic Procedures for Monomers:}

2,4-Diphenyl-quinoline (PPQ) (1), DBrFacac, 9-octyl-9-(iridium(III)bis(2,4-diphenyl-quinoline$\mathrm{N}, \mathrm{C}^{2}$ )(tridecanedionate-10,12))-2,7-dibromofluorene $\quad$ (Mon-H), bis(2,4-diphenylquinolyl$\mathrm{N}, \mathrm{C}^{2}$ )iridium(acetylacetonate) (MC-H), bis(N,N-diphenyl-N-[4-(4-phenyl-quinol-2-yl)-phenyl]amine-N, $\mathrm{C}^{3}$ )iridium(acetylacetonate) (MC-DPA), 2,7-dibromo-9,9-dioctylfluorene (5), 2,7-bis(trimethyleneborate)-9,9-dioctylfluorene (6), phosphorescent polymers (PF-H-x) appended with varying content of H-substituted phosphor (PF-H-05, PF-H-1, PF-H-2) were prepared according to previously reported procedures. ${ }^{32,} 41$ Other initial materials were bought from commercial sources and used directly. THF and toluene were processed as the routine procedures.

2-(4-bromophenyl)-4-phenylquinoline (2): 2-Aminobenzophenone (9.9 g, $50 \mathrm{mmol}$ ) and $p$ bromoacetophenone $(10.0 \mathrm{~g}, 50 \mathrm{mmol})$ were dissolved in $120 \mathrm{~mL}$ acetic acid, then $\mathrm{H}_{2} \mathrm{SO}_{4}$ (con., 4 $\mathrm{mL}$ ) was added. The mixture was cooled to room temperature (r.t.) after refluxed for $24 \mathrm{~h}$, dropped in water, then the precipitate was filtered. By recrystallization from ethanol/water, final product was obtained (12.0 g, 67\%). ${ }^{1} \mathrm{H}$ NMR (400 MHz, $\left.\mathrm{CDCl}_{3}, \mathrm{ppm}\right): \delta 7.48-7.56(\mathrm{~m}, 6 \mathrm{H}), 7.66(\mathrm{~d}, J$ $=8.4 \mathrm{~Hz}, 2 \mathrm{H}), 7.73-7.78(\mathrm{~m}, 2 \mathrm{H}), 7.91(\mathrm{~d}, J=8.4 \mathrm{~Hz}, 1 \mathrm{H}), 8.10(\mathrm{~d}, J=8.4 \mathrm{~Hz}, 2 \mathrm{H}), 8.27(\mathrm{br}, 1 \mathrm{H})$. Diphenyl-[4-(4-phenyl-quinol-2-yl)-phenyl]-phosphane oxide (3): Under an argon atmosphere, 2(4-bromophenyl)-4-phenylquinoline $2(3.6 \mathrm{~g}, 10 \mathrm{mmol})$ was disolved in $50 \mathrm{~mL}$ dry THF and cooled to $-78{ }^{\circ} \mathrm{C}$, then $5.2 \mathrm{~mL} n$-BuLi $(2.5 \mathrm{M}, 13 \mathrm{mmol})$ was added dropwisely, and stirred for another $1 \mathrm{~h}$ before addition of $2.3 \mathrm{~mL}$ chlorodiphenylphosphine. Then the reaction was warmed to r.t., quenched by water, extracted with ethylacetate and dried over anhydrous $\mathrm{Na}_{2} \mathrm{SO}_{4}$. After concentration, a mixture of $30 \mathrm{~mL}$ dichcloromethane (DCM) and $30 \mathrm{~mL} \mathrm{H}_{2} \mathrm{O}_{2}(30 \mathrm{wt} \%$ in water) was added. The reaction was kept stirring for $10 \mathrm{~h}$ at r.t., then the organic layer was separated, washed with brine solution then dried with anhydrous $\mathrm{Na}_{2} \mathrm{SO}_{4}$. The solvents was removed via 
evaporation, then the remains was purified via column chromatography on silica-gel bearing petroleum/ethylacetate $=20: 1$ as eluent to collect the ligand as a white powder $(4.3 \mathrm{~g}, 89 \%) .{ }^{1} \mathrm{H}$ NMR (400 MHz $\left., \mathrm{CDCl}_{3}, \mathrm{ppm}\right): \delta$ 7.45-7.60 (m, 12H), 7.68-7.89 (m, 8H), $7.94(\mathrm{~d}, J=8.4 \mathrm{~Hz}$, $1 \mathrm{H}), 8.30(\mathrm{~d}, J=6.9 \mathrm{~Hz}, 3 \mathrm{H})$.

N,N-Diphenyl-N-[4-(4-phenyl-quinol-2-yl)-phenyl]-amine (4): To the toluene solution, 2-(4bromophenyl)-4-phenylquinoline 2 (5.4 g, $15 \mathrm{mmol}$ ), diphenylamine (3.8 g, $22.5 \mathrm{~mol}), \mathrm{Pd}(\mathrm{OAc})_{2}$ $(100 \mathrm{mg})$ and $t$-BuONa $(2.9 \mathrm{~g}, 30 \mathrm{mmol})$ were added under argon, then $\mathrm{P}(t-\mathrm{Bu})_{3}(3.7 \mathrm{~mL})$ was added. The reaction was cooled to r.t. before reacting at $100{ }^{\circ} \mathrm{C}$ for $24 \mathrm{~h}$, dropped to water, extracted by dichcloromethane and dried over anhydrous $\mathrm{Na}_{2} \mathrm{SO}_{4}$. After removing of solvents, the residue was purified via column chromatography on silica-gel bearing petroleum/ethyacetate $=$ 30:1 as eluent, and a white powder was collected (4.8 g, 72\%). ${ }^{1} \mathrm{H}$ NMR (400 $\left.\mathrm{MHz}, \mathrm{CDCl}_{3}, \mathrm{ppm}\right)$ : $\delta 7.06(\mathrm{t}, J=7.6 \mathrm{~Hz}, 2 \mathrm{H}), 7.15-7.20(\mathrm{~m}, 6 \mathrm{H}), 7.28(\mathrm{t}, J=8.4 \mathrm{~Hz}, 4 \mathrm{H}), 7.45(\mathrm{t}, J=7.6 \mathrm{~Hz}, 1 \mathrm{H})$, 7.49-7.56 (m, 5H), 7.71 (t, $J=7.2 \mathrm{~Hz}, 1 \mathrm{H}), 7.76(\mathrm{~s}, 1 \mathrm{H}), 7.88(\mathrm{~d}, J=8.4 \mathrm{~Hz}, 1 \mathrm{H}), 8.07$ (d, $J=8$ $\mathrm{Hz}, 2 \mathrm{H}), 8.20(\mathrm{~d}, J=7.2 \mathrm{~Hz}, 1 \mathrm{H})$.

Procedures for synthesis of Ir complexes: Nonoyama method was used for the synthesis of the model and monomer Ir complexes. $\mathrm{IrCl}_{3} \cdot 3 \mathrm{H}_{2} \mathrm{O}$ (1 equiv.) together with quinoline ligand (2.5 equiv.) was added to the solvent of 2-ethoxyethanol/water $=3: 1(\mathrm{v} / \mathrm{v})$ and refluxed for $24 \mathrm{~h}$ under argon. After completion, $\mu$-chloro-bridged dimer was got through filtration. Then the dimer (1.0 mmol), DBrFacac or acetylacetone $(2.05 \mathrm{mmol})$, and sodium carbonate $(10.0 \mathrm{mmol})$ were added to the solvent of acetonitrile/chloroform ( $\mathrm{v} / \mathrm{v}=20 \mathrm{~mL} / 20 \mathrm{~mL})$, and kept reacted for $24 \mathrm{~h}$ under Ar. Upon completion, the reaction system was dropped in water, extracted with $\mathrm{CH}_{2} \mathrm{Cl}_{2}$, washed by brine and dried with anhydrous $\mathrm{Na}_{2} \mathrm{SO}_{4}$. After evaporation of the solvent, alkaline alumina column with 
the eluent of petroleum/dichloromethane from 3:1 to 2:1 was used to purify the residue and the final products were obtained.

9-octyl-9-(iridium(III)bis(diphenyl-[4-(4-phenyl-quinol-2-yl)-phenyl]-phosphane oxide$\left.N, C^{3}\right)$ (tridecanedionate-10,12))-2,7-dibromofluorene (Mon-DPO): Yield: 51\%. ${ }^{1} \mathrm{H}$ NMR (400 $\left.\mathrm{MHz}, \mathrm{CDCl}_{3}, \mathrm{ppm}\right): \delta$ 0.44-0.62 (m, 4H), 0.81-1.28 (m, 25H), $1.53(\mathrm{~m}, 3 \mathrm{H}), 1.81-1.93(\mathrm{~m}, 6 \mathrm{H})$, $4.64(\mathrm{~s}, 1 \mathrm{H}), 6.39(\mathrm{~d}, J=16 \mathrm{~Hz}, 2 \mathrm{H}), 6.93-7.24(\mathrm{~m}, 20 \mathrm{H}), 7.42-7.67(\mathrm{~m}, 22 \mathrm{H}), 7.78-7.89(\mathrm{~m}, 6 \mathrm{H})$, $8.35(\mathrm{~d}, J=12 \mathrm{~Hz}, 2 \mathrm{H}) . \quad$ MALDI-TOF $(\mathrm{m} / \mathrm{z}): 1797.4\left[\mathrm{M}^{+}\right], 1151.3\left[\mathrm{M}^{+}\right.$-DBrFacac $]$. $\mathrm{C}_{100} \mathrm{H}_{91} \mathrm{Br}_{2} \mathrm{IrN}_{2} \mathrm{O}_{4} \mathrm{P}_{2}$, Calcd: C, 66.77; H, 5.10; N, 1.56. Found: C, 66.70; H, 5.03; N, 1.47.

9-octyl-9-(iridium(III)bis(N,N-diphenyl-N-[4-(4-phenyl-quinol-2-yl)-phenyl]-amine-

$\left.N, C^{3}\right)$ (tridecanedionate-10,12))-2,7-dibromofluorene (Mon-DPA): Yield: $54 \% . \quad{ }^{1} \mathrm{H}$ NMR (400 $\left.\mathrm{MHz}, \mathrm{CDCl}_{3}, \mathrm{ppm}\right): \delta$ 0.42-0.60 (m, 4H), 0.73-1.29 (m, 25H), $1.58(\mathrm{~s}, 3 \mathrm{H}), 1.77-1.93(\mathrm{~m}, 6 \mathrm{H})$, $4.68(\mathrm{~s}, 1 \mathrm{H}), 6.25$ (dd, $J=4 \mathrm{~Hz}, 2 \mathrm{H}), 6.46-6.55(\mathrm{~m}, 2 \mathrm{H}), 6.74-6.90(\mathrm{~m}, 20 \mathrm{H}), 7.32-7.72(\mathrm{~m}, 26 \mathrm{H})$, $8.53(\mathrm{~d}, \quad J=8 \mathrm{~Hz}, 2 \mathrm{H}) . \quad$ MALDI-TOF $(\mathrm{m} / \mathrm{z}): 1732.5 \quad\left[\mathrm{M}^{+}\right], 1087.3 \quad\left[\mathrm{M}^{+}\right.$-DBrFacac $]$. $\mathrm{C}_{100} \mathrm{H}_{91} \mathrm{Br}_{2} \mathrm{IrN}_{4} \mathrm{O}_{2}$, Calcd: C, 69.31; H, 5.29; N, 3.23. Found: C, 69.26; H, 5.11; N, 3.06.

Bis(diphenyl-[4-(4-phenyl-quinol-2-yl)-phenyl]-phosphane oxide-N,C $\left.C^{3}\right)$ iridium(acetylacetonate) (MC-DPO): Yield: 48\%. ${ }^{1} \mathrm{H}$ NMR (400 MHz $\left., \mathrm{CDCl}_{3}, \mathrm{ppm}\right): \delta 1.64(\mathrm{~s}, 6 \mathrm{H}), 4.69(\mathrm{~s}, 1 \mathrm{H}), 6.38(\mathrm{~d}$, $J=20 \mathrm{~Hz}, 2 \mathrm{H}), 6.95-7.24(\mathrm{~m}, 20 \mathrm{H}), 7.36-7.65(\mathrm{~m}, 16 \mathrm{H}), 7.77-7.89(\mathrm{~m}, 6 \mathrm{H}), 8.39(\mathrm{~d}, J=8 \mathrm{~Hz}$ 2H). MALDI-TOF (m/z): 1153.2 [M $\mathrm{M}^{+}$-acac]. $\mathrm{C}_{71} \mathrm{H}_{53} \mathrm{IrN}_{2} \mathrm{O}_{4} \mathrm{P}_{2}$, Calcd: C, 68.09; H, 4.27; N, 2.24. Found: C, 67.96; H, 4.18; N, 2.19 . 